\title{
Nota Farmacológica: Corticoides intranasales para tratamiento de primera línea de la rinitis alérgica
}

Adaptado y modificado de David Stempel, MD.1

\section{Resumen}

El tratamiento de la rinitis alérgica incluye evitación de alergenos y, en algunos pacientes, medicaciones para controlar los síntomas. Los antihistamínicos son considerados por algunas guías de práctica clínica como terapia de primera línea, fundamentalmente por la ventaja percibida del comienzo de acción. Sin embargo, los antihistamínicos de primera generación son poco tolerados por sus efectos adversos centrales y los de segunda generación son relativamente costosos. Los corticoides intranasales $(\mathrm{Cl})$ han sido históricamente reservados para rinitis alérgica severa, pero últimamente están siendo ampliamente usados como tratamiento de primera línea en casos más leves. Los Cl son considerados por las guías de práctica clínica como el tratamiento sintomático más eficaz de la rinitis alérgica, con alivio de los síntomas a las 24 hs. de su inicio. Más aún, el costo del tratamiento con $\mathrm{Cl}$ es generalmente más bajo que con los antihistamínicos de segunda generación, con un muy buen perfil riesgo/beneficio. La evidencia acumulada sugiere que los $\mathrm{Cl}$ debieran ser usados como terapia de primera línea para mejorar pronóstico y reducir costos en salud. ${ }^{1}$

La rinitis alérgica es la enfermedad alérgica más común, que afecta a más de 40.000 .000 de personas en los EE.UU. Como es una entidad de poco riesgo, generalmente es subestimada por los médicos y los pacientes, quienes experimentan varios síntomas por los cuales nunca consultan. El impacto económico es considerable. En EE.UU, los costos directos de la medicación solamente en 1997 fueron 3.100 millones de dólares, incluyendo $1.600 \mathrm{mi}-$ llones para antihistamínicos no sedantes (AHNS) y 800 millones para $\mathrm{Cl}$, las visitas médicas produjeron un gasto de 1.100 millones de dólares sin contar las originadas por condiciones comórbidas como sinusitis y asma. En el Plan de Salud del Hospital Italiano de Bs. As. (con cerca de 71.000 afiliados mayores de 15 años), en los últimos 6 meses del año 2000, se recetaron el área ambulatoria alrededor de 3000 envases de corticoides intranasales que ocasionaron un gasto de $\$ 78,600$. En ese mismo período se vendieron 2323 envases de AHS que produjeron un gasto de $\$ 27.186$ y 3165 de AHNS con un gasto de 55.814 , gran parte del cual en productos combinados.?

\section{Definición}

La rinitis es una enfermedad inflamatoria de las membranas nasales causada por factores alérgicos, no alérgicos, infecciosos, hormonales, laborales, y otros. La forma más común es la alérgica, caracterizada por síntomas de congestión nasal, rinorrea, estornudos fáciles, prurito nasal, y drenaje postnasal. Puede ser desencadenada por factores estacionales (árboles, pasto, polen) o perennes (polvillo ambiental, ácaros, mohos, caspa de animales) o ambas. Los síntomas pueden persistir a lo largo del año con distinta intensidad o ser intermitentes con exacerbaciones periódicas. A su vez, puede asociarse con otras condiciones comórbidas como asma, sinusitis, pólipos nasales, y enfermedades dermatológicas.

\section{Opciones terapéuticas}

Se basan en el patrón de alergia del paciente (incluyendo estacionalidad y severidad), respuesta a la medicación, y condiciones preexistentes. Las estrategias globales son evitación de alergenos y otros desencadenantes, vacunas y alivio de los síntomas con medicaciones de venta libre, entre otros. Las dos opciones más comunes de tratamiento son los antihistamínicos orales y los $\mathrm{Cl}$. Los primeros son clasificados como de primera y segunda generación, según el potencial de efectos adversos sedantes.

\section{Antihistamínicos de primera generación}

Incluyen la clorfeniramina, difenhidramina y otros, están disponibles en los mercados desde hace muchos años. En la Argentina, la mayoría de productos que contienen estas drogas se venden en combinaciones con descongestivos nasales y son muy económicos, aproximadamente entre $\$ 7$ a 12 por mes. Son potentes antihistamínicos y pueden producir efectos sedantes o una disminución de la capacidad de funcionamiento en el 10 a $40 \%$ de los usadores, aún cuando son tomados a la hora de dormir. La evaluación de costo debe considerar los costos indirectos de los efectos adversos. Estas medicaciones no pueden ser usadas por pilotos de avión y deben administrarse con precaución en personas que manejan vehículos o máquinas.

\section{Antihistamínicos de segunda generación}

También llamados antihistamínicos no sedantes (AHNS), fueron introducidos en el mercado en 1987, comenzando con terfenadina y astemizol. Estas 2 drogas fueron removidas por asociarse a arritmias cardíacas en EE.UU. En nuestro país aún se comercializan con un precio promedio de $\$ 23$ a 29 por mes, aunque muchos de los productos existen en forma combinada para estados gripales. Las siguieron drogas AHNS como loratadina, cetirizina, fexofenadina. Tienen todos menos efectos adversos sedantes. Tienen la capacidad de reducir los estornudos, el prurito nasal y la rinorrea, así como provocar alivio parcial de la conjuntivitis alérgica, que usualmente acompaña a la rinitis. Los AHNS son relativamente costosos, entre $\$ 23$ y 38 \$ por mes en Argentina. Tienen limitada eficacia en eliminar los síntomas de congestión nasal y se asocian habitualmente con descongestivos nasales, que aumentan el costo

\section{Antihistamínicos intranasales}

El azelastine es un antihistamínico de administración nasal. Es eficaz en el tratamiento de la rinitis alérgica pero produce somnolencia en el 11,5\% de los sujetos estudiados, significativamente mayor que el placebo, por esto no se considera AHNS. En nuestro país se comercializa con un costo de $\$ 20$ mensual.

\section{Corticoides Intranasales (CI)}

Incluyen al dipropionato de beclometasona, budesonide, flunisolide, propionato de fluticasona, furoato de mometasona y acetonide triamcinolona. Estos agentes son considerados la clase de medicación más eficaz en controlar los síntomas de rinitis alérgica, de acuerdo a la reciente Guía de Tratamiento del Colegio Norteamericano de Alergia, Asma e Inmunología. ${ }^{3}$ Más allá de aliviar los síntomas de rinorrea, prurito nasal y estornudos, son eficaces en reducir la congestión nasal y, como los AHNS, son capaces de aliviar parcialmente el prurito ocular. Originalmente fueron considerados opción terapéutica para los pacientes con rinitis severa, pero están emergiendo como tratamiento de primera línea en formas más leves de rinitis alérgica por su eficacia percibida y bajo 
potencial de efectos adversos. En EE.UU, el precio de venta mensual es $25 \%$ menor que el de los AHNS. En nuestro país el precio promedio es similar al de los AHNS.

\section{Eficacia comparativa de Cl y Antihistamínicos}

Varios estudios se han conducido para saber cuáles de estos grupos producen mayor alivio a los pacientes. Esta información es de gran interés ya que los antihistamínicos son considerados de primera línea por las mismas Guías de tratamiento que consideran que los $\mathrm{Cl}$ son más eficaces en el alivio de los síntomas. La experiencia agregada a estos ensayos clínicos controlados aleatorizados ha sido sistemáticamente revisada para los $\mathrm{Cl}$ vs los antihistamínicos en general, primera y segunda generación, así como la comparación directa de $\mathrm{Cl}$ vs AHNS.

\section{CI versus antihistaminicos orales}

Weiner y cols. ${ }^{4}$ evaluaron la literatura buscando todos los trabajos aleatorizados, controlados, doble ciego, publicados entre 1966 y 1997 que compararon antihistamínicos orales con Cl en pacientes con rinitis alérgica. Identificaron 16 (2267 pacientes) y realizaron un meta-análisis. Los 16 estudios incluían antihistamínicos orales (astemizol, cetirizina, dexclorfeniramina, loratadina y terfenádina y los $\mathrm{Cl}$ beclometasona, budesonida, fluticasona y triamcinolona. Los resultados principales examinadas fueron bloqueo, congestión, descarga nasal, estonudos, prurito o molestia nasal, descarga postnasal, el total de síntomas nasales, resistencia nasal, síntomas oculares, y escalas globales de síntomas.

Los $\mathrm{Cl}$ produjeron mayor alivio significativo que los antihistamínicos orales en los siguiente síntomas: (Nota del editor: la diferencia media combinada estandarizada [DMCE] mide el tamaño del efecto en unidades de desvío estándar, los valores observados muestran efectos clínicamente significativos

- Bloqueo nasal (DMCE -0,628, IC95\% $-0,729$ a $-0,527$ )

- Estornudos (DMCE -0,488, IC $95 \%-0,588$ a $-0,387$ )

- Descarga nasal (DMCE -0,5, IC95\% -0,6 a -0,4)

- Prurito nasal (DMCE -0,38, IC95\% -0,49 a -0,21)

- Descarga postnasal (DMCE $-0,24$, IC95\% -0,42 a -0,06)

- Las DMCE entre las terapias favorecieron consistentemente a los CI. Los efectos fueron en general homogéneos entre los estudios. Se encontraron resultados similares en los nueve estudios que reportaron puntaje total de síntomas nasales. Los CI produjeron significativamente mayor alivio que los antihistamínicos orales (DMCE $-0,42$, IC95\% $-0,53$ a $-0,32$ ) pero aquí los resultados de los estudios fueron algo heterogéneos. No hubo diferencias entre los antihistamínicos orales y los $\mathrm{Cl}$ en los síntomas oculares.

\section{CI vs AHNS}

Stempel y Thomas 5 construyeron una tabia de evidencia con 13 estudios que compararon $\mathrm{Cl}$ (beclometasona, fluticasona, mometasona, o triamcinolona) versus AHNS (astemizol, loratadina o terfenadina) en pacientes con rinitis alérgica. Diez de los estudios usaron una escala de 4 puntos según gravedad de síntomas $(0=$ ninguno; 1: leve, 2:moderado y 3 : severo) mientras los restantes tres estudios usaron una escala visual analógica* de 0 (ausente) a 100 (severa). Para permitir las comparaciones de los resultados, los datos de los últimos tres estudios fueron convertidos a la escala de 4 puntos dividiéndolos por 25. Como los síntomas evaluados no fueron los mismos en los diferentes estudios, se tomó el puntaje global de síntomas, bloqueo o congestión nasal, estornudos, rinorrea y prurito nasal. Los síntomas oculares fueron evaluados en varios estudios pero el reporte fue variable. Los valores de $p$ se basaron en diferencias medias entre los tratamientos. Los Cl fueron estadísticamente superiores a los AHNS para el alivio de los síntomas nasales. La magnitud de la reducción fue de casi el doble que para los AHNS. Se observó una diferencia estadísticamente significativa a favor de $\mathrm{Cl}$ en cada uno de los estudios que evaluaron síntomas nasales y congestión nasal. Las reducciones para prurito nasal, rinorrea, y estornudos fueron mayores para $\mathrm{Cl}$ que para AHNS en todos los estudios y estadísticamente significativas en la mayoría de los estudios individuales.

Tabla.de evidencia combinada para ensayos aleatorizados de CI vs. AHNS

\begin{tabular}{|c|c|c|c|c|}
\hline Parámetro & Tratamienito: N & $\begin{array}{l}\text { Cambio } \\
\text { del hasal }\end{array}$ & $\begin{array}{c}\text { No de ECCA } \\
\text { de } 13 \text { evaluados }\end{array}$ & $\begin{array}{c}\text { ECCAA que } \\
\text { favorecen Cl* }\end{array}$ \\
\hline Sintomas totales & $\begin{array}{l}\text { Cl: } 714 \\
\text { AHNS } 752\end{array}$ & $\begin{array}{l}-5,5 \\
-3,3\end{array}$ & -1 & $8 / 8(100 \%)$ \\
\hline Congestión & $\begin{array}{l}\text { Cl: } 598 \\
\text { AHNS: } 598\end{array}$ & $\begin{array}{l}-1,1 \\
-0,6\end{array}$ & 12 & $12 / 12(100 \%)$ \\
\hline Prurito nasal & $\begin{array}{l}\text { Ĉl: } 568 \\
\text { AHNS: } 568\end{array}$ & $\begin{array}{l}-1,4 \\
-0,8\end{array}$ & 9 & $6 / 9(67 \%)$ \\
\hline Rinorrea & $\begin{array}{l}\text { Cl: } 598 \\
\text { AHNS: } 598\end{array}$ & $\begin{array}{l}-1,2 \\
-0,6 \\
\end{array}$ & 11 & $8 / 11(73 \%)$ \\
\hline Estornudos & $\begin{array}{l}\text { Cl: } 598 \\
\text { AHNS: } 598\end{array}$ & $\begin{array}{l}-1,1 \\
-0,7 \\
\end{array}$ & 11 & $8 / 11(73 \%)$ \\
\hline
\end{tabular}

*: la significancia fue definida con una $\mathrm{p}<0,05$ para la diferencia entre $\mathrm{Cl} y$ AHNS

\section{Comparaciones entre los distintos $\mathrm{CI}$}

La evidencia disponible sugiere que todos los $\mathrm{Cl}$ son comparables en eficacia. Tal es así para el alivio de los síntomas nasales de rinitis alérgica (estornudos, congestión, rinorrea y prurito) y proveen sólo alivio parcial de los síntomas oculares. Múltiples estudios "cabeza a cabeza" de diferentes $\mathrm{Cl}$, no han encontrado diferencias estadísticamente significativas o clínicamente relevantes entre los mismos. Los $\mathrm{Cl}$ se indican una o dos veces por día y han mostrado un comienzo de acción variable. Contrariamente a la percepción común, es más rápido de lo que se pensaba. Los prospectos de los fabricantes de fluticasona, mometasona y triamcinolona dicen que su comienzo de acción se produce luego de $12 \mathrm{hs}$. Se desconoce si es similar con los otros Cl. El mecanismo de este rápido comienzo es desconocido pero puede deberse al efecto vasoconstrictor local o antiinflamatorio de los corticoides. Los Cl tienen un perfil riesgo/beneficio favorable y son una alternativa segura en pacientes con rinitis alérgica. Sin embargo, mientras no se han encontrado diferencias sustanciales en eficacia, podría haberlas en el potencial de efectos adversos sistémicos. La mayoría de los $\mathrm{Cl}$ se tragan cuando se aplican vía nasal, aquellos que tienen prácticamente ninguna biodisponibilidad oral, como fluticasona y mometasona, podrían tener menores efectos sistémicos. Aunque el riesgo de efectos sistémicos a las dosis habituales es bajo, se han descripto efectos adversos relacionados a la droga en chicos con rinitis alérgica persistente tratados con beclometasona. No se han observado en cambio con mometasona nasal en rinitis alérgica ni con fluticasona inhalada oral en chicos con asma. (En la actualidad no hay ningún dato sobre efectos sobre el crecimiento con fluticasona intranasal, budesonida, flunisolida o triamcinolona). A pesar de una asociación entre cataratas y el uso de corticoides sistémicos. Derby y col no pudieron demostrar un aumento del riesgo de cataratas en usadores de $\mathrm{Cl}$ en un gran estudio observacional ${ }^{*}$ de 286.000 pacientes en el Reino Unido. 


\section{Comparaciones entre Ios AHNS}

Son similares en eficacia, tal como es observado en grandes estudios controlados.

\section{Otras comparaciones}

En un estudio se demostró que los $\mathrm{Cl}$ fueron superiores al cromolin intranasal para el alivio sintomático en alergia estacional. Más allá de este estudio la evidencia es limitada en comparaciones entre cromolin intranasal o ipratropio intranasal y $\mathrm{Cl}$ o AHNS.

\section{Modificadores de Leucotrienos}

Montelukast y zafirlukast han sido evaluados en estudios controlados para rinitis alérgica. Los datos sugieren que no tienen un rol como monoterapia para esta entidad pero pueden tener un papel adyuvante.

\section{Uso de $\mathrm{Cl}$ a demanda}

Esta forma no es adecuada para pacientes con formas moderadas a severas que requieren medicación continua. En las formas leves se prefieren los antihistamínicos orales ya que tienen un inicio de acción rápido que alivia pronto los síntomas y permite la medicación a demanda. Los más nuevos $\mathrm{Cl}$, fluticasona y mometasona han mostrado una reducción significativa de los síntomas en las primeras 12 hs de tratamiento. Un sólo estudio aleatorizado6 evaluó la administración a demanda de fluticasona 100 ug por fosa nasal por día comparada con placebo en 52 adultos con rinitis alérgica estacional. La fluticasona redujo el puntaje total de síntomas en forma significativa comparada a placebo con mejoría de la calidad de vida de los individuos. En otro estudio se ha comparado beclometasona a demanda vs beclometasona regular. La administración regular provocaba mayor beneficio que la administración a demanda. Más estudios hacen falta para comparar los efectos de la administración a demanda de CI vs los AHNS ya que es la forma más comúnmente usada por los pacientes con rinitis alérgica.

\section{Consideraciones de costo}

En la tabla de Stempel y Thomas ${ }^{5}$ se observa que para cada parámetro nasal estudiado hubo entre 57 a $100 \%$ mayor beneficio con $\mathrm{Cl}$ que con AHNS, similar a lo del estudio de Weiner ${ }^{4}$ en los síntomas oculares para lo cual son iguales $\mathrm{Cl}$ que AHNS. Los datos de seguridad parecen comparables entre los dos grupos. Dos estudios económicos comparativos norteamericanos demostraron que los $\mathrm{Cl}$ son terapias más costoefectivas. ${ }^{7-8}$

\section{Conclusiones}

El rol de los $\mathrm{Cl}$, históricamente limitado a pacientes con formas severas de rinitis alérgica está cambiando. La evidencia disponible sugiere que los $\mathrm{CI}$ no sólo son generalmente superiores a los $\mathrm{AH}$ de primera y segunda generación sino que también son una alternativa costo-efectiva en los EE.UU. con un apropiado perfil riesgo/ beneficio. En nuestro país, al ser el costo directo comparable parece razonable recomendar los $\mathrm{Cl}$ en vez de los AHNS.

\begin{tabular}{l|l|c}
\multicolumn{3}{l}{ Promedio de precios mensuales de CI y AH en Argentina } \\
\hline $\begin{array}{l}\text { Dipropionato de } \\
\text { Beclometasona }\end{array}$ & $\begin{array}{l}\text { Egosona nasal, } \\
\text { Propavent nasal }\end{array}$ & $\$ 24$ a 34 \\
\hline Budesonide & $\begin{array}{l}\text { Budeson Neumotex } \\
\text { Pulmolisoflam nasal } \\
\text { Spirocort nasal }\end{array}$ & $\$ 26-33$ \\
\hline Propionato de Fluticasona & Flixonase & $\$ 54$ \\
\hline Furoato de Mometasona & Nasonex- Uniclar & $\$ 27-26$ \\
\hline
\end{tabular}

\section{Promedio de precios AHS y NS en nuestro país}

\begin{tabular}{l|l|l}
\hline AHS & Algunos Nombres comerciales & Precios/mes \\
\hline Clorfeniramina & Decidex Isomerine & $\$ 7$ a 10 \\
\hline Difenhidramina & Benadryl & $\$ 12$ \\
\hline AHNS & & \\
\hline Terfenadina & Terfemax- Teldane & $\$ 23$ \\
\hline Astemizol & Alermizol-Vagran & $\$ 21-36$ \\
\hline Loratadina & Biloína- Clarityne & $\$ 22$ a 38 \\
\hline Cetirizina & Cetriler- Zyrtec & $\$ 29-35$ \\
\hline
\end{tabular}

\section{Comentario}

Los AHS son eficaces y muy económicos en nuestro país (aunque se debe tener en cuenta el costo indirecto de los efectos adversos) para el tratamiento de la rinitis alérgica usados como monodrogas, preparados que son raros de encontrar en el comercio ya que la mayoría de los productos tienen asociación con descongestivos nasales, corticoides, antiinflamatorios no esteroides, etc. La limitación que presentan es el alto porcentaje de efectos sedantes e incapacidad de funcionamiento que limitan su uso en la práctica cotidiana. Podríamos concluir que para aquellos pacientes en quienes el costo es un problema serio y pueden tolerar la medicación son una alternativa razonable. Si el costo directo no es un problema, los $\mathrm{Cl}$ parecieran ser las medicaciones de elección. En los casos de rinitis leve donde se desee administrar tratamiento a demanda, aunque los $\mathrm{Cl}$ parecen promisorios, existe actualmente mayor evidencia para los AHNS.

\section{Dra. Marcela Botargues [ Unidad de Medicina Familiar y Preventiva. Hospital italiano de Buenos Aires ]}

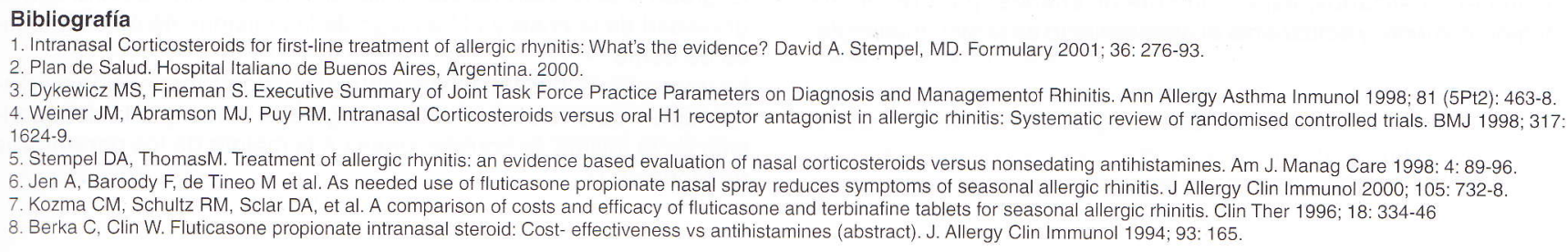

